\title{
Die liberale Theorie des Demokratischen Friedens Frieden durch weltweite Demokratisierung?
}

\author{
Constanze Scheel*
}

Abstract: This article provides an overview of the liberal democratic peace theory which holds that democracies never or almost never go to war with one another. Although this philosophical idea has circulated since Immanuel Kant, it was not scientifically evaluated until the 1960s. Nowadays it has come to be widely accepted. The US administration has expressed support for the theory above all. However, political changes in the last decade indicate that democracy will not be a universal remedy, as the example of Irak shows. The contrariness of the democratic peace remains a phenomenon: whereas members of mere democratic state groups seem to interact peacefully, they often pursue a highly aggressive foreign policy towards non-democracy nations.

Keywords: Demokratischer Frieden, Friedenspotenzial von Demokratien, Internationale Beziehungen, aktuelle Konflikte

\section{Einleitung}

Die These vom »Demokratischen Frieden« (DF-These) basiert auf Überlegungen zum Verhältnis von innerer Verfasstheit von Staaten und ihrem Außenverhalten. Die Debatte um den »Demokratischen Frieden« dreht sich konkret um die Frage, ob Demokratien friedensfähiger und friedfertiger sind als andere Staatsformen und ergo keine oder fast keine Kriege gegen andere Demokratien führen. Ist das politische System der Demokratie intrinsisch friedensstiftend? Bringt die »friedliche innere Verfasstheit auch nach außen Frieden? Diese Fragen werden von Vertretern des Liberalismus bejaht, was wiederum einen optimistischen Blick in die Zukunft eröffnet. Im vorliegenden Aufsatz soll der Frage nachgegangen werden, ob dieser Optimismus gerechtfertigt ist und inwiefern der These des »Demokratischen Friedens« eine Differenzierung ansteht.

\section{Die Idee hinter der Theorie}

Obgleich bereits im antiken Griechenland über das Verhältnis von Demokratie und Frieden nachgedacht wurde, stammt der einflussreichste Klassiker zum Thema des »Demokratischen Friedens« aus dem Zeitalter der Aufklärung, und zwar von Immanuel Kant, der - neben anderen Philosophen seiner Zeit oder der Zeit vor ihm wie Abbé de Saint-Pierre und JeanJacques Rousseau - damit begann, Voraussetzungen und Bedingungen eines stabilen Friedens zu untersuchen und eine Friedenstheorie formulierte. ${ }^{1}$

Kant war der Erste, der einen Zusammenhang zwischen Republiken und Frieden postulierte und 1795 in seiner Abhandlung »Zum ewigen Frieden« eine revolutionäre These aufstellte, die gegenwärtig immer wieder ins Zentrum der politischen Diskussionen rückt. Peter Graf Kielmannsegg, emeritierter Professor für Politikwissenschaft in Mannheim, formuliert den Kern des »politischen Vermächtnisses « Immanuel Kants folgendermaßen: »Nur Demokratien seien auf Dauer friedensfähig. Die

\footnotetext{
* Constanze Scheel (Maitrîse LEA - Université de Nice) ist als Sprachwissenschaftlerin bei der Bundeswehr in Strausberg tätig und forscht seit $2004 \mathrm{zu}$ friedenswissenschaftlichen Themen am Institut Frieden und Demokratie der FernUniversität Hagen.

1 Lutz Schrader: Frieden und Demokratie, 2005, S. 15.
}

Friedensfähigkeit und Friedensbereitschaft eines Staates wird von seiner inneren Verfassung bestimmt. $\ll^{2}$

Dieses Theorem ist auf seine politische Tauglichkeit hin zu überprüfen. Insbesondere gilt es, die Frage zu untersuchen, ob der alte Menschheitstraum vom (ewigen) Frieden durch weltweite Demokratisierung realisiert werden kann. In jedem Falle wäre die Aufklärung des Zusammenhangs von politischer Herrschaftsform und Kriegswahrscheinlichkeit, bzw. Friedensaussichten in den Staatenbeziehungen in ihrer theoretischen und politischen Tragweite kaum zu überschätzen. ${ }^{3}$

Heute wie damals geht es um dieselben Fragen: Ist die innere, demokratische Verfasstheit eines Staates ausschlaggebend für die Friedensbereitschaft nach außen (und übrigens auch nach innen)? Wie sehen die Kriterien dafür aus?

\section{Das liberale Konzept des »Demokratischen Friedens«}

\subsection{Der »Demokratische Frieden« - ein empirisches Gesetz?}

In Politik und Wissenschaft ist die These weit verbreitet, dass die demokratische Verfasstheit eines Staates sein außenpolitisches Verhalten in Richtung einer friedlichen Politik beeinflusst. Die Behauptung einer (prinzipiellen) Friedfertigkeit demokratischer Staaten ist in zahlreiche Regierungserklärungen sowie in außen- und sicherheitspolitische Konzepte und Strategiepapiere eingeflossen und wird von der offiziellen Politik demokratischer Staaten gerne als Tatsache dargestellt. ${ }^{4}$

Arend Wellmann stellt fest: »In der friedenswissenschaftlichen Diskussion wird heute überwiegend die Auffassung vertreten, dass die These vom »Demokratischen Frieden« einem sozial-

\footnotetext{
2 Vgl. Peter Graf Kielmannsegg: Drei Regeln für den Frieden, Politik Essay, in: Die Zeit Nr. 53 vom 22. Dezember 2004.

3 Lutz Schrader: Frieden und Demokratie, 2005, S. 23.

4 So formuliert Peter Schlotter, Vorsitzender der Arbeitsgemeinschaft für Friedens- und Konfliktforschung (AFK) die Fragestellung für das AFK-Kolloquium 2003 mit dem Thema »Demokratien im Krieg«, vgl. http://www.afk-web. de/html/2003.html.
} 
wissenschaftlichen Gesetz nahe komme. $\ll^{5}$ Auch Teusch und Kahl merken an, dass kaum eine politikwissenschaftliche These wie die vom »Demokratischen Frieden « eine vergleichbar große Zahl an empirischen und theoretischen Arbeiten hervorgebracht hat. ${ }^{6}$ Oft ist gar die Rede von einem empirischen Gesetzesrang, den die DF-These durch den überzeugenden Nachweis einer praktischen Abwesenheit von Kriegen zwischen demokratischen Staaten einnehmen würde. ${ }^{7}$

Miriam F. Elman würdigt ebenso die Abwesenheit von Krieg zwischen Demokratien und die Theorie, die diesen »demokratischen Frieden « erklärt, als »einen der wichtigsten empirischen Befunde und Theorien, die aus dem Studium der internationalen Beziehungen in den vergangenen Jahrzehnten hervorgegangen sind . $^{8}$

Da diese These von der liberalen Schule in den Internationalen Beziehungen hervorgebracht wurde, triumphieren die Verfechter des (Neo-)Liberalismus nun über die (Neo-)Realisten, die die Möglichkeit dauerhafter und verlässlicher friedlicher Beziehungen zwischen den Staaten prinzipiell ausschließen. ${ }^{9}$

\subsection{Liberale Erklärungsansätze}

Welche Erklärungen hält die Theorie bereit, diese Erkenntnis zu untermauern? Manche Forscher sehen die Strukturen und Institutionen innerhalb demokratischer Staaten als entscheidenden Faktor an, andere begründen die Theorie eher normativ.

Zum Verständnis des Phänomens des »Demokratischen Friedens« lassen sich drei Erklärungsansätze bzw. »Perspektiven« innerhalb der liberalen Theorie ausmachen, wie Joachim Krause erklärt: »(1) solche, die institutionelle Arrangements von Demokratien in den Vordergrund stellen (institutionalistische Perspektive), (2) solche, die die für Demokratien typischen Normen und Werte verantwortlich machen (normativ-kulturelle Perspektive) sowie (3) solche, die einen Zusammenhang herstellen zwischen dem Verhalten von demokratischen Staaten und der Perzeption anderer als ebenfalls >demokratisch (konstruktivistische Perspektive) «. ${ }^{10}$

Der erste Erklärungsansatz baut darauf auf, dass Demokratien institutionelle Barrieren gegen Machtmissbrauch haben, die politische Entscheidungsprozesse schwerfällig und komplex machen und somit kriegerisches Verhalten erschweren oder gar unmöglich machen. Hier wird insbesondere auf die Gewaltenteilung abgestellt, die als wesentlicher Faktor der Zivilisierung des Verhaltens von Demokratien dargestellt wird. ${ }^{11}$

5 Arend Wellmann: Zur Diskussion um den demokratischen Frieden, in: Wissenschaft und Frieden 3-98.

6 Ulrich Teusch/Martin Kahl: Ein Theorem mit Verfallsdatum? Der »Demokratische Frieden« im Kontext der Globalisierung, in: Zeitschrift für Internationale Beziehungen, 2001, S. 287.

7 Ebda., S.288.

8 Miriam F. Elman: Paths to Peace. Is Democracy the Answer? Cambridge, 1997, S.1.

9 Lutz Schrader: Frieden und Demokratie, 2005, S. 24 f.

10 Joachim Krause: Vom ewigen Frieden zum demokratischen Frieden? Die Aussagekraft von Theorien des demokratischen Friedens, in: Zeitschrift für Politikwissenschaft 2/2003, S. 696.

11 Bruce Bueno de Mesquita/David Lalman: War and Reason. Domestic and International Imperatives, 1992, Kap.4
Die normativ-kulturelle Perspektive geht davon aus, dass Demokratien spezifische Normen entwickeln, die sie dazu bringen, friedlicher in der Außenpolitik aufzutreten. »Die Normen des regulierten politischen Wettbewerbs, die Kompromisslösung politischer Konflikte und der friedlichen Machtübergabe werden von Demokratien externalisiert, wenn sie es mit anderen nationalen Akteuren in der Weltpolitik zu tun haben. «12

Zu der konstruktivistischen Perspektive heißt es: Demokratien führen deshalb keinen Krieg gegeneinander, weil sie sich als artverwandt und prinzipiell sympathisch ansehen. Dabei spielen gemeinsame Normen und artverwandte Strukturen zwar eine Rolle, entscheidend ist aber, wie sich Großgesellschaften gegenseitig perzipieren. ${ }^{13}$

Die drei unterschiedlichen Erklärungsansätze schließen sich nicht gegenseitig aus, sondern sollen sich vielmehr in der Erklärung eines bestimmten Phänomens unterstützen.

Die Empirie scheint also ein friedlicheres Zusammenleben von Demokratien zu zeigen, woraus die optimistische Hoffnung (der Vertreter des Liberalismus) formuliert wird - mathematisch ausgedrückt -: je mehr Demokratien (im internationalen Staatensystem) - je mehr Frieden (in der Welt).

\subsection{Genesis und Entwicklung der neueren theoretischen Debatte}

Der demokratische Frieden - definiert als Abwesenheit von Kriegen zwischen den demokratischen westlichen Staaten - ist eine Realität. ${ }^{14}$ Diese Aussage führt die Wissenschaft zu der spannenden Frage der Beweiskraft. Die Theorie des »Demokratischen Friedens « stellt insofern ein Paradoxon dar, als demokratische Staatsformen in Frieden mit anderen Demokratien leben und somit den (realen) Endzustand des alten Menschheitstraums vom ewigen Frieden erreicht haben.

Nun stellt sich die Frage, worauf sich dieses Theorem gründet bzw. wie dieses Phänomen theoretisch erklärt wird. Kants Entwurf einer friedlichen Welt (Zum Ewigen Frieden, 1795) basiert auf der These, dass die Friedensfähigkeit und Friedensbereitschaft eines Staates von seiner inneren Verfassung bestimmt wird und dass nur eine Welt von Republiken eine friedliche Welt sein kann. Mit Republik meint Kant, in unserer Begrifflichkeit, die verfassungsstaatliche, gewaltenteilende, repräsentative Demokratie. ${ }^{15}$

Die Debatte zur Kausalität von Demokratie und Frieden nimmt ihren Anfang in den 1960er Jahren, wobei dort zunächst die

12 Bruce Russett: Grasping the Democratic Peace. Principles for a Post-Cold War World, 1993, S. 33. Michael W. Doyle: Liberalism and World Politics, in: American Political Science Review 80/1986, S. 1151-1169.

13 Colin Kahl: Constructing a Separate Peace: Constructivism, Collective Liberal Identity and Democratic Peace, in: Security Studies 8/1998-1999, S. 94

Thomas Risse-Kappen: Democratic Peace - Warlike Democracies? A Social Constructivist Approach, in: European Journal of International Relations 1/1995, S.491-517.

14 Joachim Krause: Vom ewigen Frieden zum demokratischen Frieden? Die Aussagekraft von Theorien des demokratischen Friedens, in: Zeitschrift für Politikwissenschaft 2/2003, S. 693.

15 Vgl. Peter Graf Kielmannsegg: Drei Regeln für den Frieden, Politik Essay, in: Die Zeit Nr. 53 vom 22. Dezember 2004. 
Konflikt- und Gewaltträchtigkeit demokratischer und auch anderer Staatsformen im Vordergrund stand. ${ }^{16}$

Als »Geburtsurkunde« der Erforschung des »Demokratischen Friedens « gilt Dean Babsts erster Aufriss der These des »Demokratischen Friedens «, erschienen 1964 unter dem Titel »Elective Governments - A Force for Peace« im »Wisconsin Sociologist $« .{ }^{17}$ Der Kriminologe Babst kommt zu dem Schluss, dass »zwischen 1789 und 1941 keine Kriege zwischen unabhängigen Nationen mit gewählter Regierung ausgefochten wurden ${ }^{1}{ }^{18}$

Doch dieser fernab des wissenschaftlichen und publizistischen Mainstreams erschienene Beitrag erhielt erst mit seiner Entdeckung im Jahre 1972 durch Melvin Small und J. David Singer seine Bedeutung, die in ihrem Aufsatz auf der Grundlage aufwändiger empirischer Erhebungen zu sämtlichen Kriegen zwischen 1816 und 1965 ebenso feststellten, dass Demokratien im untersuchten Zeitraum keine Kriege gegeneinander geführt haben. Dies führten sie jedoch auf die Tatsache zurück, dass besagte Demokratien »selten Nachbarn gewesen sind « (» Krieg ist am wahrscheinlichsten zwischen Nachbarn $\ll) .{ }^{19}$ Des Weiteren stellten sie aber auch fest, dass Demokratien im Zeitraum zwischen 1816 und 1965 ebenso oft an internationalen Kriegen beteiligt waren wie nicht-demokratische Staaten.

In den darauffolgenden Jahren wurde die Babst'sche Hypothese nicht weiter verfolgt. Erst Rudolph J. Rummel legt in seinem fünfbändigen Werk »Understanding Conflict and War « (1975-1981) eine fundierte Argumentation zu den Ursachen von Konflikt und Krieg vor, in dem das Friedenspotenzial von Demokratien eine zentrale These war. ${ }^{20}$ Insgesamt formuliert er dreiunddreißig Hypothesen des »Demokratischen Friedens«, aus denen er folgende Erkenntnis zieht: »libertarian states have natural inhibitions on involvement in violence: the responsiveness of elected leaders to domestic interest groups or public opinion, which ordinarily will oppose violence, tax increases and conscription. $^{21}$ Darüber hinaus gebe es zwischen freiheitlichen Staaten »eine fundamentale Sympathie ihrer Völker gegenüber dem jeweils anderen System, eine Vereinbarkeit der grundlegenden Werte, grenzüberschreitende Druckausübung und sich überlappende Gruppen und Organisationen und die Ausbreitung von Macht und Interessen.«

Auch Rummels Arbeit wird anfänglich nicht zur Kenntnis genommen, bis er im Jahre 1983 seinen Aufsatz »Libertarianism and International Violence ${ }^{22}$ veröffentlichte und die These vertrat: je »freiheitlicher «bzw. demokratischer ein Staat ist, desto gewaltaverser verhält er sich in seinen Außenbeziehungen. In seinem Aufsatz hat Rummel anhand empirischer Analysen versucht, die Existenz des »liberalen Friedens« nachzuweisen. Die Debatte um den »demokratischen Frieden« ging

16 Vgl. insbesondere Michael Haas: Societal Approaches to the Study of War, in: Journal of Peace Research 4/1965, S. 307-323.

17 Dean V. Babst: Elective Governments - A Force for Peace, in: The Wisconsin Sociologist $1 / 1964$, S. 9-14.

18 Ebda., S. 10.

19 Melvin Small/J. David Singer: The War Proneness of Democratic Regimes, in: Jerusalem Journal of International Relations 4/1976, S. 50-69.

20 Vgl. Rudolph J. Rummel: Understanding Conflict and War, Vol. 1 - 5, erschienen zwischen 1975 und 1981.

21 Ebd., Vol. 4, 1979, S. 292.

22 Rudolph J. Rummel: Libertarianism and International Violence, in: Journal of Conflict Resolution 2/1983, S. 27-71. mit diesem Text in die zweite Runde, die bis heute anhält. In Erweiterung der oben erwähnten These von Babst vertritt Rummel die Annahme, dass Demokratien grundsätzlich immer friedfertiger sind als andere, was als monadische Theorie bezeichnet wird. ${ }^{23}$ Diese ist jedoch in der Wissenschaft sehr umstritten, weil sie zu radikal scheint.

Die bei Russett ${ }^{24}$ angelegte dyadische Theorie ${ }^{25}$ des »Demokratischen Friedens « gilt unter Vertretern des liberalen Ansatzes heute als vorherrschend. Sie stellt das politische System und die politische Kultur als bestimmende Faktoren der Außenpolitik dar. Demokratien unterliegen demnach Gewaltbeschränkungen, die durch ihre innere Verfasstheit verursacht werden. Sie führen also gegeneinander keinen Krieg, dagegen sind Kriege zwischen Demokratien und autokratisch verfassten Staaten aber möglich.

In verschiedenen wissenschaftlichen Projekten ${ }^{26}$ wurden allerdings empirische Daten zu bilateralen Staatenbeziehungen ausgewertet und mit quantitativen Verfahren qualitative Aussagen getroffen, worin eine große Schwäche der Theorie des »Demokratischen Friedens « liegt. Russett hat die Ergebnisse dieser Untersuchungen in seinem Buch »Grasping the Democratic Peace « folgendermaßen zusammengefasst: 1. Es sei praktisch unmöglich, für die Zeit seit 1815 einen einzigen Krieg zwischen zwei Demokratien zu identifizieren; 2. Demokratien unterscheiden sich in ihrer generellen Bereitschaft zum Krieg und zur bewaffneten Intervention nicht grundsätzlich von anderen Systemen; daher seien Kriege, an denen Demokratien beteiligt waren, keine Seltenheit, selbst solche, bei denen die Demokratien Angreifer waren.

\section{Der empirische Doppelbefund der DF-Theorie}

Mit seinem in der Fachzeitschrift »Philosophy and Public Affairs « veröffentlichten zweiteiligen Artikel wird schließlich Michael W. Doyle ${ }^{27}$ ein weiterer Protagonist in der theoretischen Debatte um den »Demokratischen Frieden«. Doyle hat die Ergebnisse der bisherigen Forschungen und eigener Arbeiten erstmalig resümiert und verhalf der Theorie des »Demokratischen Friedens « Anfang der 1980er Jahre zum Durchbruch in der modernen IB-Forschung (Internationale Beziehungen). Er stellt insbesondere die Doppelgesichtigkeit des »liberalen Friedens « heraus: auf der einen Seite der Frieden zwischen den demokratischen Staaten (in seiner ganzen politischen Tragweite) und auf der anderen Seite die Konflikt- und Kriegsneigung der demokratischen Staaten gegenüber nicht-demokratischen Staatsformen. Diese beiden Seiten des außenpolitischen Verhaltens bezeichnet Doyle als das »liberale Erbe « und formuliert damit den Forschungsstand, der bis heute vielfach als der

23 Joachim Krause: Vom ewigen Frieden zum demokratischen Frieden? Die Aussagekraft von Theorien des demokratischen Friedens, in: Zeitschrift für Politikwissenschaft 2/2003, S. 698.

24 Vgl. Bruce Russett: Grasping the Democratic Peace. Principles for a Post-Cold War World, 1993.

25 In den Sozialwissenschaften wird der Terminus Dyaden für bilaterale Staatenbeziehungen verwendet.

26 Beispielsweise Daten des »Correlates of War«-Projekts der University of Michigan und des »International Crisis Behavior «-Projekts von Michael Brecher und Jonathan Wilkenfeld.

27 Michael W. Doyle: Kant, Liberal Legacies, and Foreign Affairs, in: Philosophy and Public Affairs 3/1983, S. 205-235 und 4/1983, S. 323-353. 
Quasi-Konsens (»near-consensus«) unter den Anhängern der »democratic peace proposition « bezeichnet wird. ${ }^{28}$

Die Tatsache, dass Demokratien im internationalen Staatensystem proportional genauso oft in Kriege verwickelt werden wie Nicht-Demokratien - nur eben nicht untereinander -, hat sich als »empirischer Doppelbefund « in der DF-Forschung niedergeschlagen und harrt nach wie vor einer überzeugenden Erklärung. ${ }^{29}$

Ebenso müssen die Untersuchungen zur Frage, was Demokratien veranlasst, sich gegenüber Nicht-Demokratien nicht friedlich zu verhalten, noch ausgeweitet werden.

Zusammenfassend lässt sich also festhalten: Es existiert auf der einen Seite eine Zone des Friedens zwischen demokratischen Staaten und auf der anderen Seite eine Zone des Unfriedens zwischen Demokratien und Nicht-Demokratien, zu der auch die Beziehungen von Nicht-Demokratien untereinander gehören. ${ }^{30}$

Diese Erkenntnis steht weiterhin im Mittelpunkt der Forschungen zum Phänomen des »Demokratischen Friedens «. Vom »Schattengewächs « oder »Stiefkind « der US-amerikanischen wissenschaftlichen Debatte in den 1960er Jahren wird die Theorie des »Demokratischen Friedens « heute im Zusammenhang mit fast jedem aktuellen Konflikt diskutiert (Afghanistan, Irak, ...), insbesondere natürlich bei Konflikten, an denen die Vereinigten Staaten von Amerika (als Begründer dieser Theorie und als größter Demokratie-Exporteur) beteiligt sind.

Trotz der quasi empirischen Geltung unter Sozialwissenschaftlern weisen Kritiker auf teilweise große Schwachstellen der DF-Theorie hin.

\section{Die Schwachstellen der Theorie des »Demokra- tischen Friedens«}

\subsection{Kritik an der Methodik}

Eine große Schwäche der Theorie des »Demokratischen Friedens« liegt darin, dass mit quantitativen Untersuchungen qualitative Aussagen getroffen wurden, bzw. werden. Dabei spielen natürlich die Variablen Demokratie und Krieg bzw. Frieden eine große Rolle. Die Ergebnisse variieren entsprechend der jeweiligen Definition von Demokratie und / oder Krieg. Als Definition von Demokratie wurde beispielsweise von Bruce Russett zugrundegelegt:

»For modern states, democracy is usually identified with a voting franchise for a substantial fraction of citizens, a government brought to power in contested elections, and an executive either popularly elected or responsible to an elected

28 Nils Petter Gleditsch: Democracy and Peace, in: Journal of Peace Research 4/1992, S. 369 f.

29 Errol A. Henderson: Democracy and War - The End of an Illusion, 2002, S. 5. 30 Carsten Rauch: Die Theorie des Demokratischen Friedens, 2005, S. 24. legislature, often also with requirements for civil liberties such as free speech. $\ll^{31}$

Und von Sontheimer:

»Sie (die Prinzipien der westlichen Demokratie/Anm. der Verfasserin) lassen sich in folgenden Merkmalen zusammenfassen: $\mathrm{Zu}$ einer Demokratie in unserem Sinne gehören die politische Meinungs- und Organisationsfreiheit der Bürger, die Bildung mehrerer, miteinander konkurrierender politischer Parteien und das Recht auf Opposition, die periodische Abhaltung von demokratischen Wahlen der Staatsbürger, die auch einen Wechsel der Regierung nach sich ziehen können. Dies alles ist gebunden an eine rechtsstaatliche Ordnung und ihre Verfahren, deren oberster Wert die Achtung der Würde des Menschen und daraus abgeleiteter Grundrechte ist. ${ }^{32}$

Bei der Minimaldefinition nach Schmidt ${ }^{33}$ wird unter Demokratie ein politisches System verstanden, »in dem das Volk seine entscheidungsbefugten Führungen frei aus miteinander konkurrierenden Gruppen und Individuen, die nicht von der Regierung bestimmt wurden, auswählt «.

Unterschiedliche Forscher setzen unterschiedliche Schwerpunkte bei der Definition von »Demokratie« und generell herrscht keine Übereinstimmung in diesem Bereich. Was dazu führt, dass man - je nach Definition der Variablen - die Zahl der Fälle (in denen von friedlichen oder kriegführenden Demokratien die Rede ist) massiv beeinflussen und somit das Ergebnis immens variieren kann. Da diese Variable (übrigens genauso wie die Variable Krieg) sehr komplex ist, sind viele Forscher dazu übergegangen, den einzelnen Ländern einen bestimmten Demokratisierungsgrad aufgrund eines Demokratie-Indizes ${ }^{34}$ zuzuweisen. Dies relativiert den Vorwurf der fehlerhaften Methodik. Nichtsdestoweniger bleibt er weiterhin bestehen.

\subsection{Kritik an der Statistik}

Nicht zuletzt wird von den Kritikern der Theorie die statistische Signifikanz des Befundes nicht akzeptiert. John Mearsheimer, der freilich zu den Vertretern des Realismus in den Internationalen Beziehungen zählt, stellt fest:

»(D)emocracies have been few in number over the past two centuries, and thus there have not been many cases where two democracies were in a position to fight with each other. « ${ }^{35}$

Es gab also bisher zu wenige Demokratien und noch weniger Demokratien, die Krieg gegeneinander führten. Dies verleitet zu der nahe liegenden Annahme, dass die Korrelation zwischen Demokratie und Frieden lediglich auf den Zufall zurückzuführen ist bzw. ein Epiphänomen ist.

31 Bruce Russett: The Fact of Democratic Peace, in: Brown Michael et al.: Debating the Democratic Peace - An International Security Reader, 1996, S. $58-81$.

32 Kurt Sontheimer: So war Deutschland nie - Anmerkung zur politischen Kultur der Bundesrepublik, 1999, S. 173.

33 Manfred G. Schmidt: Demokratietheorie. Eine Einführung, 2000, S. 409.

34 Zwei der bekanntesten Demokratie-Indizes sind der »Freedom House «-Index und der Polity-II (bzw. III)-Datensatz von Ted Gurr. Vgl. Freedom House (1990 ff) und Jaggers / Gurr (1995).

35 John J. Mearsheimer: Back to the Future: Instability in Europe after the Cold War, in: International Security 1/1990, S. 50 
Dasselbe Definitionsproblem stellt sich bei der Variablen Krieg. Eigentlich müsste ja die Variable Frieden zugrundegelegt werden, doch aus praktischen Gründen wird zumeist auf die Variable Krieg zurückgegriffen, für die im Allgemeinen die Definition von Small/Singer und ihrem $»$ Correlates of War«-Projekt benutzt wird. ${ }^{36}$ Bei einem Konflikt handelt es sich demnach dann um einen Krieg, wenn mindestens 1000 Kombattanten zu Tode gekommen sind.

Diese Definition hat jedoch den Schwachpunkt, dass die rasante Entwicklung in der Militärtechnologie (Luftkriege, Electronic Warfare, etc) nicht berücksichtigt ist; sie kann dazu führen, dass Bedenken gegen Kriege nachlassen - die »High Tech«-Kriege wären kurz und »sauber « und fordern wenig Opfer.

Diese Definition hat jedoch den Schwachpunkt, dass sie die Realitäten moderner Kriegführung außer Acht lässt. Die Fixierung auf Kombattanten kann dazu führen, dass Bedenken gegen Kriege nachlassen und den Glauben erzeugen, »High Tech«-Kriege seien kurz, sauber und forderten wenige Opfer.

Denn der Verwendung des Begriffs Kombattanten liegt die Definition von Krieg als organisierte Form der Gewalt zugrunde. So stellt sich die Frage, ob diese Einschränkung auf Kombattanten noch zeitgemäß ist. Antwort: Sicherlich ist sie dies nicht. Hier ist das Beispiel des Kosovo-Einsatzes der NATO gegen Jugoslawien zu nennen, in dem die Anzahl der toten Soldaten auf Seiten der NATO deutlich unter 1000 lag. ${ }^{37}$

Die vorgelegten Zahlen sind daher immer nur in Abhängigkeit der vom jeweiligen Forscher bzw. im Rahmen des jeweiligen Forschungsprojekts gewählten Definitionen zu sehen.

\section{Zusammenfassung und Ausblick}

Demokratien führen so gut wie keine Kriege gegeneinander. Dies ist der empirische Befund der Theorie des »Demokratischen Friedens« und man möchte nur allzu gern an ihn glauben. Dann wären »wir im Westen« auf dem richtigen Weg mit unseren Demokratisierungsstrategien bzw. unserem Demokratisierungseifer.

Doch ein Blick in die Tageszeitungen und - zur vertiefenden Kenntnis - auf das Konfliktbarometer des Heidelberger Insti-

\footnotetext{
36 Vgl. Melvin Small / David J. Singer: Resort to Arms: International and Civil Wars, 1816-1980, 1982

37 Vgl. Carsten Rauch: Die Theorie des Demokratischen Friedens, 2005, S. 27, Fußnote 93.
}

tuts für Internationale Konfliktforschung ${ }^{38}$ zeigt ein anderes Bild. Im Jahr 2006 werden 278 politische Konflikte gezählt. Sechs davon sind Kriege und 29 ernste Krisen; damit werden insgesamt 35 Konflikte mit massivem Gewalteinsatz ausgetragen. 83 Konflikte werden als Krisen klassifiziert, was bedeutet, dass nur gelegentlich Gewalt eingesetzt wird. Insgesamt werden 118 Konflikte mit Gewalteinsatz ausgetragen. Dagegen gibt es 160 gewaltlose Konflikte, die in 100 manifeste und 60 latente Konflikte unterschieden werden können. Ein Vergleich zwischen den Jahren 2005 und 2006 ergibt folgendes Bild:

Im Vergleich zum letzten Jahr erhöht sich die Anzahl der Konflikte, die auf der höchsten Intensitätsstufe ausgefochten werden, signifikant von zwei auf sechs Kriege. Diese sechs Konflikte sind Somalia (verschiedene Rebellengruppen), Sudan (Darfur), Sri Lanka (LTTE), Afghanistan (Taliban), Irak (Rebellen) und Israel (Hisbollah). Zwei dieser Kriege, im Sudan (Darfur) und der Konflikt im Irak (Aufständische) sind auch im Vorjahr, wie bereits 2004, auf derselben Intensitätsstufe geführt worden. Von den vier Kriegen, die 2005 nicht auf dieser höchsten Intensitätsstufe ausgetragen wurden, waren die in Afghanistan (Taliban) und in Israel (Hisbollah) im Jahre 2005 als ernste Krisen eingestuft worden. Im Vergleich dazu verliefen die Konflikte in Somalia (mehrere Rebellengruppen) und in Sri Lanka (LTTE) 2005 mit einem relativ geringen Ausmaß an Gewalt. Die Anzahl ernster Krisen erhöht sich 2006 leicht von 26 auf 29. Insgesamt steigt die Anzahl von Konflikten auf den zwei höchsten Intensitätsstufen von 28 im Jahre 2005, der niedrigsten Anzahl seit Jahrzehnten, auf 35 im Jahre 2006. Die Anzahl der Krisen, die Konflikte mittlerer Intensität darstellen, ist leicht zurückgegangen - von der seit 1945 höchsten Anzahl von $90 \mathrm{im} \mathrm{Jahr} 2005$ auf 83 im Jahr 2006. Während dies einerseits auf Deeskalationsprozesse zurückzuführen ist, erkennt man andererseits, dass die sehr hohe Anzahl von Krisen 2005 ein klar erkennbares Warnsignal gewesen ist. Sie hatte

38 Vgl. dazu ausführlicher http://www.hiik.de/konfliktbarometer/index.html.de. 
angedeutet, dass die niedrigste Zahl hochgewaltsamer Konflikte aller Zeiten im Jahr 2005 nicht der Beginn eines Trends hin zu einer friedlicheren Welt gewesen ist, da acht Krisen in diesem Jahr zu hochgewaltsamen Konflikten eskalierten.

Aufgrund der unzähligen Regime im Wandel und bisher nicht erfolgter Klassifizierung der hier dargestellten Länder in einer einheitlichen Demokratisierungsskala, kann hier keine Unterscheidung zwischen Demokratien und Nicht-Demokratien gemacht werden. Dennoch wird anhand der Graphik deutlich, dass es trotz aller Bemühungen der »führenden « westlichen Demokratien, ihr Modell zu verbreiten, nicht gelungen ist, die Welt friedlicher zu gestalten.

Die eingangs aufgeworfene Frage nach der Rechtfertigung der optimistischen Sichtweise der Vertreter der liberalen Schule, nach der die »friedliche « innere Verfasstheit von politischen Systemen auch friedensbringend nach außen wirke, also auf globaler Ebene, muss anhand dieser Analyse grundsätzlich verneint werden.

Sicher sind demokratische Normen und Strukturen intrinsisch friedlich, d.h. gewaltlos. Aber - so stellt Arend Wellmann fest - die Gleichsetzung von Demokratie und Frieden bleibt weit hinter dem Wissen um friedensfähige inner- und zwischengesellschaftliche Strukturen zurück. ${ }^{39}$

Lutz Schrader weist zu Recht darauf hin, dass von der Fähigkeit der westlichen Demokratien zum inneren Wandel in den kommenden Jahren in hohem Maße die Dynamik und Friedensverträglichkeit des »democratic enlargement « abhängen wird. Denn - so die herrschende Ansicht - die weltweite Ausbreitung von Demokratie und Frieden vollzieht sich in erster Linie über die Beispielwirkung der bereits existierenden demokratischen Staaten. ${ }^{40}$

39 Arend Wellmann: Zur Diskussion um den demokratischen Frieden, in: Wissenschaft und Frieden 3/1998.

40 Lutz Schrader: Frieden und Demokratie, 2005, S. 203.
Harald Müller greift Bedenken zum »Demokratischen Frieden $«$ in seinem Aufsatz »Arroganz der Demokratien ${ }^{41}$ auf. Dort heißt es: Unter praktischen Gesichtspunkten habe sich der wissenschaftliche Diskurs über den »Demokratischen Frieden « negativ auf die Politik ausgewirkt. »Demokratie« sei im Verlauf der 1990er Jahre zum Kriterium der Unterscheidung zwischen Freund und Feind geworden, sie habe die besten Chancen zur moralischen Selbstrechtfertigung geboten. Angesichts der großen globalen Herausforderungen (etwa auf dem Gebiet der Umwelt, der Demographie, des Terrorismus, der ungleichen Verteilung von Ressourcen und des Zugangs zum Markt, etc.) ist es allerdings kontraproduktiv, die Welt in »Gut« (westliche Demokratien) und »Böse« (Rest der Welt) einteilen zu wollen. Vielmehr muss eine (weitere) Sensibilisierung für übergeordnete Interessen erfolgen. In dieser Hinsicht haben die USA jedoch versagt (man denke beispielsweise an das Kyoto-Protokoll). In diese Bresche könnte die Europäische Union springen, um ihre Position in der Welt zu stärken.

Die politische Entwicklung der letzten Jahre zeigt, dass Demokratie kein Allheilmittel ist. In einer kritischen Bilanz merkt Lutz Schrader an: »Auf der internationalen Ebene werden die etablierten demokratischen Staaten, und insbesondere das politische System der USA, zum Maßstab dessen erhoben, was als demokratisch zu gelten hat. ${ }^{42}$ In gewissen Kreisen (insbesondere in US-amerikanischen Regierungskreisen) wird das Seelenheil der (bisher anti-demokratischen) Restwelt mittels des universalen (westlichen Demokratie-) Modells propagiert.

Angesichts dieser Entwicklungen möchte man zu mehr Selbstreflexion und zu mehr Bescheidenheit aufrufen. Möglicherweise hätte man den Irak vor einem Bürgerkrieg bewahren können, wenn mehr Rücksicht geübt und mehr Zeit in den Demokratisierungsprozess investiert worden wäre.

41 Harald Müller: Die Arroganz der Demokratien - Der »Demokratische Frieden « und sein bleibendes Rätsel, in: Wissenschaft und Frieden 2/2003. Dieser Aufsatz entstand im Rahmen des seit Juli 2000 laufenden Forschungsprogramms der Hessischen Stiftung Friedens- und Konfliktforschung (HSFK) "Antinomien des demokratischen Friedens", in dessen Rahmen die Widersprüchlichkeiten und Anomalien der Theorie in zahlreichen Projekten untersucht werden.

42 Lutz Schrader: Frieden und Demokratie, 2005, S. 203. 